Dominique Labie Jacques Elion

\title{
Modulation polygénique des maladies monogéniques: l'exemple de la drépanocytose
}

La drépanocytose résulte d'une mutation unique et son mécanisme physiopathologique a été minutieusement exploré; elle constitue donc un modèle de choix pour aborder le problème de la variabilité d'expression clinique des maladies monogéniques. Peuvent être la cible de phénomènes modificateurs: (1) la polymérisation de l'hémoglobine $S$ (diminuée par l'hémoglobine fotale); (2) la concentration intra-érythrocytaire en hémoglobine $S$, si existe une $\alpha$-thalassémie concomitante, ou une modification de l'action de facteurs transrégulateurs sur l'expression respective des

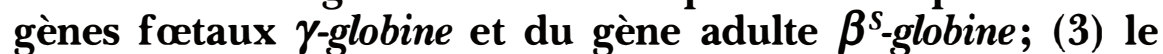
profil particulier d'expression de protéines autres que l'hémoglobine dans les réticulocytes de drépanocytaires, car la drépanocytose est aussi une maladie cellulaire et rhéologique: anomalies des constituants de la membrane érythrocytaire et de sa perméabilité, phénomènes d'adhérence aux parois vasculaires, activation de la cascade de la coagulation, sont tous des processus faisant intervenir des protéines partenaires qui sont, ou pourraient être, polymorphes.

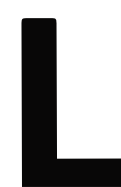

a drépanocytose, première maladie moléculaire identifiée, la plus explorée sans doute, reste le sujet de nombreux travaux. De par sa fréquence et sa gravité, elle est dans certains pays un lourd problème de santé publique, pour lequel plusieurs approches thérapeutiques sont l'objet de recherches intenses. Sur le plan théorique, la question persiste d'une discordance entre la précision des connaissances acquises sur l'hémoglobine $\mathrm{S}(\mathrm{HbS})$ et l'extrême variabilité d'expression clinique de la maladie. Depuis 1949, on a défini successivement la substitution protéique en cause, la mutation responsable au niveau de l'ADN, toujours la même, une transversion $\mathrm{A} \rightarrow \mathrm{T}$ au niveau du sixième codon du gène $\beta$-globine, le processus supramoléculaire de polymérisation de l'HbS sous l'influence d'une baisse de la pression en oxygène, et ses conséquences en terme de déformation et de fragilisation de l'érythrocyte [1]. Ce schéma physiopathologique simple n'explique ce- 


\section{RÉFÉRENCES}

1. Noguchi CT, Schechter AN, Rodgers GP. Sickle cell disease pathophysiology. In: Higgs D, Weatherall DJ, eds. The haemoglobinopathies. Bailliere's Clinical Haematology, vol. 6. London: Baillière Tindall, 1993: 57 91 .

2. Rosa J. Un modèle en recherche clinique: la drépanocytose. médecine/sciences $1986 ; 2: 558-67$.

3. Serjeant GR. The clinical features of sickle cell disease. In : Higgs D, Weatherall DJ; eds. The Haemoglobinopathies. Bailliere's Clinical Haematology, vol. 6. London: Bailliere Tindall, 1993: 93-115.

4. Bodmer J, Cavalli-Sforza LL. Genetics, evolution and man. San Francisco: Freeman, 1976.

5. Lehmann H, Huntsman RG. Man's haemoglobins. Amsterdam, Oxford: North Holland Publishing Company, 1966.

6. Perrine RP, Pembrey ME, John P, Perrine $\mathrm{S}$, Shoup F. Natural history of sickle cell anemia in Saudi Arabs. Ann Intern Med $1978 ; 88: 1-6$.

7. Brittenham G, Lozoff B, Harris JW, Sharma VS, Narasimhan S. Sickle cell anemia and trait in a population of Southern India. Am J Hematol 1977; 2 : 25-32.

8. Kan YM, Dozy AM. Polymorphism of DNA sequence adjacent to human $\beta$-globin structural gene: relationship to sickle mutation. Proc Natl Acad Sci USA, 1978; 75 : 56315.

9. Pagnier J, Mears JG, Dunda-Belkhodja O, Schaefer-Rego K, Beldjord C, Nagel RI., Labie $D$. Evidence for the multicentric origin of the sickle hemoglobin gene in Africa. Proc Natl Acad Sci USA 1984;81 : 1771-3.

10. Kulozik AE, Wainscoat JS, Serjeant GR, Kar BC, Al-Awamy B, Essan GJF, Falusi AG, Haque SK, Hilali AM, Kate S, Rasaninghe WAEP, Weatherall DJ. Geographical survey

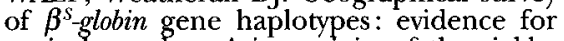
an independent Asian origin of the sicklecell mutation. Am J Hum Genet 1986; 9 : 239 44.

11. Lapouméroulie C, Dunda $\mathrm{O}$, Ducrocq R, Trabuchet G, Mony-Lobé M, Bodo JM, Carnevale P, Labie D, Elion J, Krishnamoorthy $R$. A novel sickle cell mutation of yet another origin in Africa: the Cameroon type. Hum Genet 1992 ; 89: 333-7.

12. Berg PE, Mittelman M, Elion J, Labie D, Schechter AN. Increased protein binding to a -530 mutation of the human $\beta$-globin gene associated with decreased $\beta$-globin synthesis. Am J Hematol 1991; 36: 42-7. pendant que partiellement la maladie observée. Elle présente en effet un large spectre d'associations diverses: anémie hémolytique, crises vaso-occlusives douloureuses aux localisations multiples, accidents obstructifs graves des gros vaisseaux, épisodes infectieux, puis ulcères de jambe, et complications chroniques osseuses et viscérales [2, 3]. Certains de ces symptômes sont, certes, en relation directe avec le désordre moléculaire et la déformation érythrocytaire; d'autres, en revanche, sont largement rhéologiques et font intervenir des mécanismes plus complexes qui ont, eux aussi, été explorés au cours des dernières décennies. C'est ainsi qu'ont pu être mises en évidence certaines données physiopathologiques qui sont autant de pistes de recherche. (1) D'autres hémoglobines, présentes dans le globule rouge, peuvent avoir un effet bénéfique, ce qui est vrai en particulier de l'hémoglobine fœtale ( $\mathrm{HbF})$, ou au contraire délétère. (2)

La concentration intra-érythrocytaire de l'hémoglobine est un facteur majeur de gravité, et la maladie est atténuée par tout ce qui réduit cette concentration, exacerbée au contraire par tous les phénomènes de déshydratation. 3) Enfin, parce qu'il y a hémolyse, la durée de vie des globules rouges est brève; la réticulocytose est constante, modifiant le profil d'expression habituellement observé dans les cellules érythroïdes des protéines autres que l'hémoglobine; cela explique sans doute l'adhérence cellulaire accrue à l'endothélium vasculaire. On est donc amené à considérer deux types de facteurs: (1) corpusculaires, concernant l'hémoglobine et le globule rouge en général, dans leurs composantes moléculaires et cellulaires et (2) extracorpusculaires, concernant le globule rouge drépanocytaire dans son environnement. Les recherches fondamentales et les essais thérapeutiques sont partis de ces constatations. Les études les plus poussées ont été menées sur les populations drépanocytaires panmictiques du continent américain, aux États-Unis ou aux Antilles, parce que la maladie y était fréquente et que les moyens d'exploration disponibles permettaient la recherche. Le polymorphisme d'expression de la maladie suggère une modulation génétique multifactorielle.
Histoire naturelle et premières explorations

Très tôt, parallèlement aux travaux poursuivis en pays d'immigration, Lehmann recueillait des observations concernant l'histoire naturelle de la maladie sur les lieux, et donc dans les populations, de sa diffusion originelle. L'Afrique tropicale, du Sahara au Zambèze, est la zone d'incidence maximale. Les études épidémiologiques permettent aussi d'y dessiner des foyers de concentration majoritaire [4] (figure 1). La maladie a également été décrite dans des zones très éloignées les unes des autres, parmi les tribus du sous-continent indien, autour du golfe Persique et en Iran. Enfin, des foyers disséminés, en Afrique du Nord et en différents points du Bassin méditerranéen, sont bien connus. Très anciennement déjà, dans le contexte de leur histoire naturelle, l'observation de séries de malades mettait en évidence des différences de gravité indubitables d'une région à l'autre [5]. En Afrique centrale, il était rare qu'un enfant drépanocytaire survive à ses premières années, si bien que certaines études épidémiologiques réalisées chez des adultes avaient conclu initialement à l'absence ou à la rareté de la mutation dans certaines régions! En Afrique de l'Ouest, en revanche, on a toujours vu des patients atteignant l'âge adulte et présentant les manifestations classiques et les complications invalidantes de la maladie. Dans le golfe Persique, c'est chez des sujets actifs et ayant mené une vie presque normale que le diagnostic de drépanocytose a pu être posé au cours d'enquêtes systématiques [6]. De même, l'étude de foyers de drépanocytose dans les tribus du Sud de l'Inde démontrait une survie normale des adultes, puisque la proportion observée des homozygotes et des hétérozygotes respectait celle attendue selon la loi de HardyWeinberg, malgré des conditions socio-économiques et de médicalisation comparables à celles observées en Afrique [7]. Cette gamme de gravité, s'étendant des cas mortels dans la première enfance à d'autres dont le diagnostic n'est fait qu'après plusieurs décennies de vie normale, alors que la mutation causale est toujours la même, suggère évidemment l'implication de facteurs différents de cet- 


\section{RÉFÉRENCES}

13. Elion J, Berg PE, Lapouméroulie C, Trabuchet G, Mittelman M, Krishnamoorthy R, Schechter AN, Labie D. DNA sequence variation in a negative control region 5 ' to the $\beta$-globin gene correlates with the phenotypic expression of the $\beta^{s}$ mutation. Blood 1992; 79: 787-92.

14. Talbot D, Philipsen S, Fraser P, Grosveld F. Detailed analysis of the site 3 region of the human $\beta$-globin dominant control region. $E M B O J$ f $990 ; 9: 2169-78$.

15. Liu D, Chang JC, Moi P, Liu W, Kan YW, Curtin PT. Dissection of the enhancer activity of $\beta$-globin 5 ' DNase I-hypersensitive site 2 in transgenic mice. Proc Natl Acad Sci USA 1992; 89: 3899-903.

16. Périchon B, Ragusa A, Lapouméroulie C, Romand A, Moi P, Ikuta T, Labie D, Elion J, Krishnamoorthy R. Inter-ethnic polymorphism of the $\beta$-globin gene locus control region (LCR) in sickle-cell anemia patients. Hum Genet 1993; 91 : 464-8.

17. Powers PA, Smithies O. Short gene conversions in the human fetal globin gene region; a by-product of chromosome pairing during meiosis. Genetics 1986; 112: 34358 .

18. Ragusa A, Lapouméroulie C, Lombardo M, Elion J, Labie D, Krishnamoorthy R. Differential binding of nuclear protein factors to the polymorphic ${ }^{\wedge} \gamma$ IVS2 (TG) repeat region and its relevance to $\mathrm{HbF}$ response in stress anemia. Blood 1992; 80 (suppl 1): 80 a.

19. Donovan-Peluso M, Acuto S, O'Neill D, Kaysen J, Hom A, Maggio A, Bank A. The regulation of $\gamma$-globin gene expression. Ann NY Acad Sci 1990; 612: 160-5.

20. Sampietro M, Thein SL, Contreras M, Pazmany L. Variation of $\mathrm{Hb} \mathrm{F}$ and F-cell number with the G- $\gamma$ XmnI (C-T) polvmorphism in normal individuals. Blood 1992; phism in

21. Bouhassira E, Nagel RL. A 6-bp deletion 5 ' to the ${ }^{\text {' }} \gamma$ globin gene in $\beta^{S}$ chromosomes bearing the Bantu haplotype. Hum Genet $1990 ; 47: 161-3$.

22. Beldjord C, Ducrocq R, Nadifi S, Lapoumeroulie C, Elion J, Labie D. A haplotypelinked four base pair deletion upstream of the ${ }^{A} \gamma$ globin gene coincides with decreased gene expression. Hum Genet 1992; 89: 625-8.

te seule mutation. On sait que les conditions culturelles, socio-économiques, ou de prise en charge médicale sont importantes. Elles ne sauraient tout expliquer sans l'intervention de facteurs génétiques. Origine unicentrique ou pluricentrique de la maladie, des discussions en sont longtemps restées à justifier par l'anthropologie l'une ou l'autre des hypothèses.
Une avancée fondamentale a été la démonstration que la mutation drépanocytaire existait dans des contextes chromosomiques variables. En 1978, Kan et Dozy décrivaient le polymorphisme de restriction (restriction fragment length polymorphism, RFLP) dans l'ADN humain [8]. Le premier site identifié, reconnu par l'enzyme HpaI, était en déséquilibre de liaison avec la mutation drépanocytaire. La 


\section{RÉFÉRENCES}

23. Pissard S, Beuzard Y. A potential regulatory region for the expression of fetal hemoglobin in sickle cell disease. Blood 1994 ; 84: $331-8$

24. Ragusa A, Lapouméroulie C, Lombardo M, Beldjord C, Lombardo T, Nagel RL, Labie D, Krishnamoorthy R. Nucleotide variation in the 3' $y$ enhancer region are linked to $\beta$-gene cluster haplotypes and are unrelated to fetal hemoglobin expression. $A m J$ Hum Genet 1989; 45: 106-11

25. Embury SH, Steinberg MH. Genetic modulators of disease. In Embury SH, Hebbel RP, Mohandas N, Steinberg MH, eds. Sickle cell disease. Basic principles and clinical practice. New York: Raven Press, 1994: 279-98.

26. Kulozik A, Kar BC, Serjeant GR, Serjeant BE, Weatherall DJ. The molecular basis of $\alpha$ thalassemia in India. Its interaction with the sickle cell gene. Blood 1988; 71 : 467-72.

27. Labie D, Srinivas R, Dunda O, Dodé C, Lapouméroulie C, Devi V, Devi S, Ramasami K, Elion J, Ducrocq R, Krishnamoorthy $\mathrm{R}$, Nagel RL. Haplotypes in tribal Indians bearing the sickle cell gene: evidence for the unicentric origin of the $\beta^{\mathrm{s}}$ mutation and the unicentric origin of the tribal populations of India. Hum Biol 1989; 61 : 479-91.

28. Mukherjee MB, Lu CY, Ducrocq R, Gangakhedakar RN, Colah RB, Kadam MD, Mohanty D, Nagel RL, Krishnamoorthy R. Sickle cell anemia linked to Arab-India haplotype in two populations with different levels of alpha thalassemia. 20th Annual meeting of the national sickle cell disease program. Boston march 18-21, 1995; p. 208.

29. Christakis J, Vavatsi N, Hassapopoulou H, Papadopoulou M, Mandraveli K, Loukopoulos D, Morris JS, Serjeant BE, Serjeant GR. Comparison of homozygous sickle cell disease in Northern Greece and Jamaica. Lancet 1990 ; 335 : 637-40.

30. Miyoshi K, Kaneto Y, Kawai H, Ohchi H, Niki S, Hasegawa K, Shirakami A, Yamano T. X-linked dominant control of F-cells in normal adult life: characterization of the Swiss type as hereditary persistence of fetal hemoglobin regulated dominantly by gene(s) on X chromosome. Blood 1988; 72 : 1854-60.

31. Dover GJ, Smith KD, Chang YC, Purvis S, Mays A, Meyers DA, Sheils C, Serieant G. Fetal hemoglobin levels in sickle cell disease and normal individuals are partially controlled by an X-linked gene located at Xp22.2.

même approche, utilisant onze sites de restrictions répartis sur l'ensemble $\mathrm{du}$ locus $\beta$-globine, permettait, peu après, de montrer que la mutation $\beta^{\mathrm{s}}$ se retrouve en Afrique liée à trois haplotypes de restriction différents [9]. Chacun de ces haplotypes est spécifique d'un foyer géographique, Afrique occidentale, golfe du Bénin et Afrique centrale, et on l'y retrouve de façon presque homogène. L'environnement chromosomique chez les sujets arabo-indiens, est différent [10]; enfin, un foyer minoritaire a été récemment retrouvé au Cameroun [11]. Ces résultats sont en faveur d'une origine multicentrique $\left(\mathrm{m} / \mathrm{s}, n^{\circ} 1\right.$, vol. 3, p.54), mais l'éventualité d'une origine unique, suivie de remaniements chromosomiques complexes, ne peut être complètement écartée. Il reste que la mutation a évolué dans un contexte génétique différent dont on peut faire l'hypothèse qu'il participe à la variabilité d'expression de la maladie. En effet, le locus $\beta$, sur le chromosome 11 , contient les divers gènes de globine exprimés aux différentes étapes du développement, dont les gènes fotaux $\gamma$. Or on sait que l'HbF interrompt la croissance du polymère d'HbS et, par là même, exerce un effet atténuateur sur la maladie. Des variations de séquence dans le locus $\beta$ globine, en cis de la mutation $\beta$, pourraient donc être susceptibles de modifier l'équilibre d'expression entre les gènes $\beta$ et $\gamma$ globine et donc le rapport $\mathrm{HbF} / \mathrm{HbS}$.

La polymérisation de l'HbS est, par ailleurs, extrêmement sensible à des variations même minimes de sa concentration intra-érythrocytaire. Les $\alpha$-thalassémies, le plus souvent délétionnelles, ont été sélectionnées au cours de l'histoire, comme la drépanocytose, par le paludisme à Plasmodium falciparum; elles coexistent souvent dans les mêmes populations $\left(\mathrm{m} / \mathrm{s} n^{\circ} 8-9\right.$, vol. 10, p. 905). Associées à la drépanocytose, elles peuvent, en diminuant la concentration intra-érythrocytaire d'HbS, constituer un facteur modulateur en trans susceptible d'en moduler l'expression clinique.

Nous rappellerons d'abord ce qu'on sait actuellement concernant les deux éléments majeurs que sont (1) les polymorphismes du locus $\beta$-globine et leur lien avec la régulation de l'expression relative des gènes $\gamma$ et $\beta$, d'une part, et (2) l'influence des $\alpha$ thalassémies, d'autre part. Nous signalerons ensuite dans quelles directions s'orientent des travaux à la recherche d'autres déterminants génétiques susceptibles de moduler l'expression de la maladie drépanocytaire.

\section{Maladie drépanocytaire et polymorphismes du locus $\beta$-globine}

Les haplotypes de restriction tels que décrits initialement sont des marqueurs dont l'association à un taux plus ou moins élevé d'HbF, établi chez des homozygotes de populations ethniquement homogènes, n'est que statistique. Depuis leur description, il y a plus de 10 ans, on a toujours insisté sur le nombre important d'exceptions à ces données moyennes. Deux séries de développements ont fait récemment progresser les connaissances. D'une part, grâce aux études sur systèmes cellulaires et souris transgéniques, on connaît mieux les éléments de séquence qui participent, soit à proximité des gènes eux-mêmes, soit à distance, dans la région majeure de régulation du locus (LCR), à la régulation de la transcription des gènes globine au cours du développement. De même, des avancées décisives ont été réalisées dans la connaissance des facteurs protéiques de régulation se fixant sur ces éléments. D'autre part, l'avènement de l'amplification in vitro de l'ADN par PCR a permis d'identifier de nombreux polymorphismes nouveaux dont certains situés dans des segments d'ADN fonctionnellement importants. Ces polymorphismes sont de types variés: mutations ponctuelles, microdélétions, mais aussi répétitions en nombre variable de courtes séquences nucléotidiques ou microsatellites. Bien que la liste n'en soit pas exhaustive, trois d'entre elles méritent une mention particulière, car elles semblent impliquées dans une modulation de l'équilibre relatif d'expression des gènes fœetaux et adultes, et, à ce titre, pourraient moduler la gravité clinique de la maladie drépanocytaire:

1. A $0,5 \mathrm{~kb}$ en 5 ' du gène $\beta$-globine, il existe une zone de régulation négative, de type $(\mathrm{AT})_{x} \mathrm{~T}_{y}$, qui fixe un facteur nucléaire BP1 [12]. Le couple 


\section{RÉEÉRENCES}

32. Chang YC, Smith KD, Moore RD, Serjeant GR, Dover GJ. An analysis of fetal hemoglobin variation in sickle cell disease the relative contributions of the X-linked factor, $\beta$-globin haplotypes, $\alpha$-globin gene number, gender, and age. Blood 1995;85: 1111-7.

33. Thein SL, Sampietro M, Rohde K, Rochette J, Weatherall DJ, Lathrop GM, Demenais $F$. Detection of a major gene for heterocellular hereditary persistence of fetal hemoglobin after accounting for genetic modifiers. Am J Hum Genet 1994 ; 54 : 214-28.

34. Craig JE, Rochette J, Fisher C, Weatherall DJ, Marc S, Lathrop GM, Demenais F, Thein SL. Dissecting the loci controlling fetal haemoglobin production on chromosomes $11 \mathrm{p}$ and $6 \mathrm{~g}$ by the regressive approach. Nature Genet 1996; 12 ; 58-64.

35. Hebbel RP. Beyond hemoglobin poly merization: the red blood cell membrane and sickle disease pathophysiology. Blood $1991 ; 77: 21437$.

36. Ciu D, Lubin B, Eoelofsen B, Van Deenen L. Sickled erythrocytes accelerate clot ting in vitro: an effect of abnormal membrane lipid asymmetry. Blood 1981; 58: 398-401.

37. Mohandas N, Evans E. Rheological and adherence properties of sickle cells. Potential contribution to hematological manifestations of the disease. Ann NY Acad $S_{c i}$ $1989 ; 565: 327-37$

38. Gee BE, Platt OS. Sickle reticulocyte adhere to VCAM-1. Blood 1995; 85: 268-74.

39. Francis RB Jr, Johnson CS. Vascular occlusion in sickle cell disease: curren concepts and unanswered questions. Blood 1991; 77 : 1405-14.

40. Smolinski PA, Offermann MK, Eckman JR, Wick TM. Double-stranded RNA induces sickle erythrocyte adherence to endothelium: a potential role for viral infection in vaso-occlusive pain episodes in sickle cell anemia. Blood 1995; 85 : 2945-50.

41. Agre P. The aquaporin water channels 20th Annual meeting of the national sickle cell disease program. Boston, march 18-21, 1995.

$\mathrm{m} / \mathrm{s}^{\circ} 3$, vol. 12 , mars 96 $x / y$ est en liaison stricte avec chacun des haplotypes qui caractérisent les diverses origines géographiques de la mutation $\beta^{s}$. Les résultats obtenus in vitro concernant la fixation de BP1 sont corroborés par les taux d'expression observés in vivo chez des cohortes de sujets $A S$ d'origine géographique et ethnique définie: une corrélation inverse est retrouvée entre l'affinité du facteur BP1 et la biosynthèse de la chaîne $\beta$ anormale. Ainsi, la séquence liée à l'haplotype arabo-indien fixe BP1 avec la plus forte affinité, et chez les sujets porteurs de cet haplotype, la synthèse de la chaîne $\beta^{s}$ est très faible par rapport à celle observée chez les sujets africains [13].

2. Dans le LCR lui-même, un microsatellite de type $(\mathrm{AT})_{x} \mathrm{~N}_{12}(\mathrm{AT})_{y}$ a été décrit au niveau du site hypersensible HS2 [14]. Une dissection de l'activité de HS2 a montré que ce microsatellite, situé dans la moitié 3 ' de la séquence active, exerce une régulation négative sur l'activateur majeur, site de fixation pour les facteurs AP-1/NF-E2, situé dans la moitié 5' de la même séquence [15]. Chez des sujets $S S$ d'origine géographique déterminée et homozygotes pour les haplotypes de restriction spécifiques de chaque région, une spécificité du couple $x / y$ a également été retrouvée [16]. Ces différentes séquences fixent aussi un facteur nucléaire; la compétition observée entre la fixation sur ce microsatellite en HS2 et sur celui qui existe en 5 ' du gène $\beta$ fait supposer qu'il s'agirait du même facteur BP1 ou d'un facteur de la même famille protéique. Sachant qu'il existe aussi des sites de fixation pour BP1 (non polymorphes) en amont des gènes $\gamma$, des modèles sont concevables qui pourraient participer, au moins partiellement, à une modification de l'équilibre d'expression relatif en gènes fœtaux et adultes selon l'affinité des différents motifs polymorphes pour ce facteur de régulation négative. Plusieurs fois déjà, nous avons mentionné l'existence d'un tel équilibre. Chez un individu donné, la production totale de chaînes $\beta$ et $\gamma$ globine est constante et tout facteur modifiant la production de l'une retentit en sens inverse sur la production de l'autre. Par exemple, dans les cas de persistence héréditaire d'Hb F (PHHF) dues à une mutation ponctuelle dans un des promoteurs $\gamma$, la surexpression du gène $\gamma$ correspondant s'accompagne d'une diminution équivalente de l'expression du gène $\beta$, alors que ce dernier est strictement normal. Les drépanocytaires indiens qui expriment le moins leur gène $\beta^{s}$, parce qu'ils présentent une fixation accrue de BP1 en amont du gène $\beta$, sont ceux aussi qui expriment au plus haut niveau leurs gènes $\gamma$.

3. Au niveau des gènes $\gamma$ globine deux approches ont attiré l'attention sur le deuxième intron (IVS2). Tout d'abord, par clonage et séquençage de plusieurs gènes ${ }^{G} \gamma$ et ${ }^{A} \gamma$, le groupe de Smithies avait noté l'existence d'un polymorphisme de type (TG) dans la partie 3' de l'intron [17]; la variété des motifs s'est accrue récemment par l'exploration de sujets présentant un taux particulièrement élevé d'HbF [18]. Sur les chromosomes drépanocytaires, c'est au niveau du gène ${ }^{A} \gamma$ que le degré de polymorphisme est le plus élevé. Par ailleurs, en utilisant des constructions de gènes chimères $\beta /{ }^{A} \gamma$, transfectés de façon stable dans des cellules K562, on avait pu montrer le rôle de IVS2 au cours de l'induction de la synthèse par l'hémine [19]. Des résultats encore partiels semblent montrer que les différents motifs identifiés fixent de façon variable un facteur nucléaire et pourraient donc moduler de façon différentielle la synthèse d'HbF induite par l'anémie. Le seul RFLP auquel on ait attribué une signification fonctionnelle correspond à un polymorphisme $\mathrm{C} \rightarrow \mathrm{T}$ situé en position -158 de la coiffe ${ }^{G} \gamma$ et exploré par l'enzyme XmnI. On a souvent constaté que la présence d'un $\mathrm{T}$ en -158 (XmnIt) était associée à un taux élevé d'HbF en cas d'anémie; de façon constante, elle est associée à une expression prépondérante de la chaîne ${ }^{\mathrm{G}} \gamma$ [20]. Cependant, un rôle direct de la séquence contenant ce polymorphisme n'a jamais été démontré in vitro. De façon intéressante, la présence du site $X m n I$ est strictement liée à un motif particulier $(T G)_{13}$, au niveau de l'IVS2 du gène ${ }^{A} \gamma$. Il est tentant de penser que le site $X m n I$ n'est qu'un marqueur, la régulation de synthèse s'exerçant au niveau des introns polymorphes. On ne peut que citer aussi d'autres polymorphismes du locus $\gamma$-globine, qui 


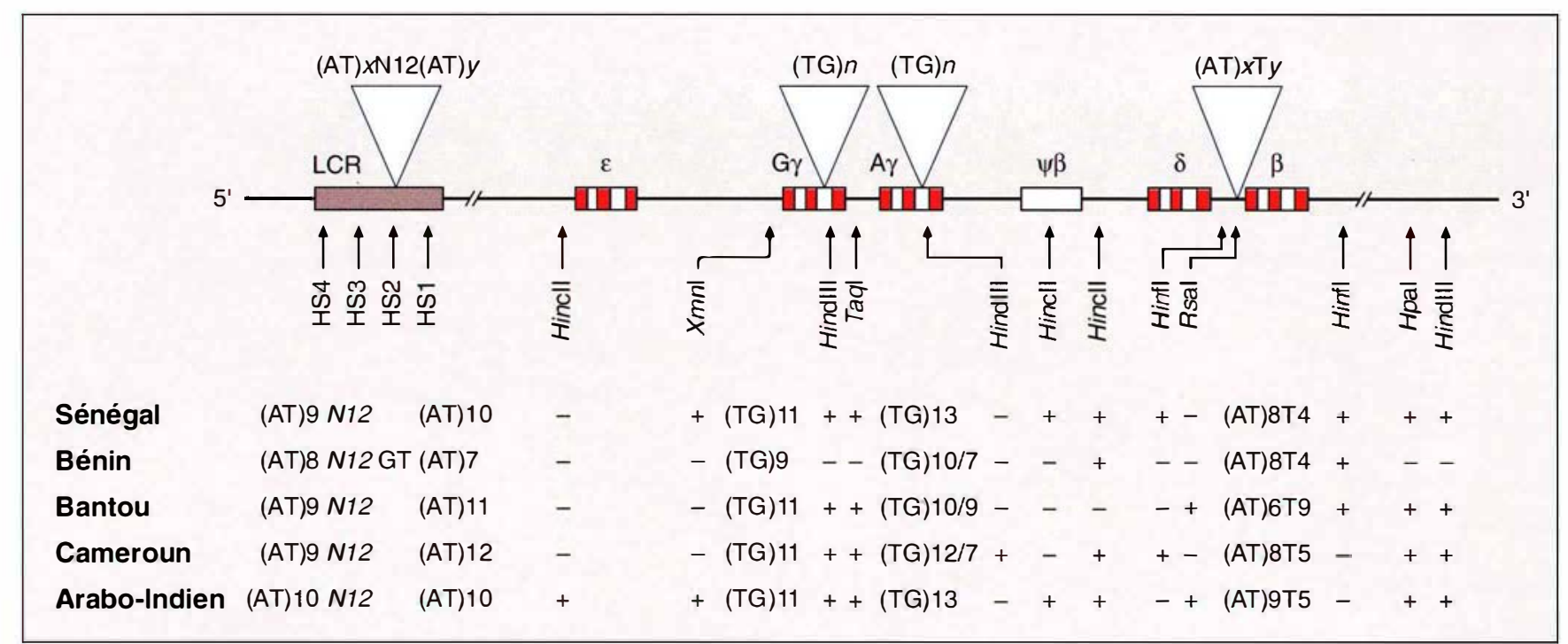

Figure 2. Haplotypes étendus des chromosomes drépanocytaires. Dans ces haplotypes sont figurés: (1) les 12 sites de restriction polymorphes (+ ou -) informatifs dans les populations drépanocytaires; (2) les motifs polymorphes de séquences répétées dont la valeur fonctionnelle semble établie dans la région de contrôle du locus (LCR), dans les IVS2 des gènes $\gamma$ et en amont du gène $\beta$.

n’ont pas donné lieu à l'heure actuelle à une caractérisation fonctionnelle: courtes délétions en 5' du gène ${ }^{G} \gamma$ chez les drépanocytaires de type Bantous [21], en $5^{\prime}$ du gène ${ }^{A} \gamma$ chez ceux du Cameroun [22] ; mutations ponctuelles dans la région pré${ }^{G} \gamma$ [23]; mutations en 3 ' du gène ${ }^{A} \gamma$ dans la région enhancer [24]... L'ensemble de ces polymorphismes dont la liste est loin d'être limitative permet actuellement de définir des «haplotypes étendus " (figure 2), très variables sur les chromosomes portant le gène globine $\beta^{4}$, mais qui semblent stables sur $100 \mathrm{~kb}$ quand on explore les chromosomes drépanocytaires d'origine ethnique homogène chez lesquels a joué la sélection par le Plasmodium falciparum. Si certaines corrélations entre génotype et phénotype semblent exister dans ces groupes homogènes, il reste très ambigu d'apprécier le rôle spécifique de tel ou tel élément dans des populations plus mélangées, surtout si on n'explore pas la totalité du locus $\beta$ globine. En effet, il est aléatoire d'apprécier le rôle de chacun des allèles chez les hétérozygotes composites; il faut aussi s'assurer de l'absence de recombinaison entre les différents c'est non chacun d'eux, mais leur combinatoire, qui agit sur la régulation d'expression des différents gènes du locus.

\section{Maladie drépanocytaire et $\alpha$-thalassémie}

On sait que la mutation $\beta^{s}$ et les $\alpha$ thalassémies ont été soumises à la même sélection par le paludisme. Il n'est donc pas étonnant de retrouver ces deux types de défauts moléculaires dans les mêmes populations, et donc des individus ayant cohérité des deux défauts. En diminuant la concentration corpusculaire en hémoglobine (CCMH), toute $\alpha$-thalassémie tend à réduire la polymérisation de l'HbS. C'est donc, a priori, un premier facteur d'atténuation en trans, même si des études cliniques ont pu démontrer l'association à certaines complications spécifiques [25]. Les études épidémiologiques, tout en confirmant la fréquence de l'hétérozygotie pour l' $\alpha$-thalassémie délétionnelle $(\alpha \alpha /-\alpha)$, ont révélé des incidences très variables: $\approx 30 \%$ dans les populations noires immigrées en Amérique, de même qu'en Afrique occidentale, $10 \%$ seulement au Sénégal, $50 \%$ ou même davantage dans les populations Bantous d'Afrique centrale. La même proportion de $50 \%$ est observée chez les drépanocytaires d'Arabie saoudite. En Inde, où des groupes ethniques très endogames ont été explorés, la proportion de drépanocytaires $\alpha$-thalassémiques varie d'un groupe à l'autre. Elle a été trouvée chez plus de $60 \%$ des sujets SS dans une étude faite en Orissa (fréquence génique $-\alpha /: 0,32$ ) [26]. Dans quelques groupes tribaux du Sud de l'Inde ou du Gujerat, cette fréquence atteint la quasi-fixation, entre $90 \%$ et $100 \%$, mais se retrouve également dans la population témoin non drépanocytaire [27]. Une étude récente a permis de comparer deux groupes de drépanocytaires indiens, tribaux et urbains de basse caste. La fréquence génique $\beta^{S}$ était similaire $(0,22$ et 0,23$)$, les taux d'HbF comparables $(18,5$ et $15,5 \%)$, aucune différence n'a été retrouvée au niveau du locus $\beta$-globine. Seule différait la fréquence de l' $\alpha$-thalassémie, 0,97 chez les tribaux, et 0,24 chez les urbains. La maladie se présentait comme beaucoup plus sévère dans le second groupe que dans le premier, démontrant clairement le rôle modulateur du nombre de gènes $\alpha$ actifs comme élément de gravité [28]. 


\section{RÉFÉRENCES}

42. Dahlbäck B, Carlsson M, Svensson PJ Familial thrombophilia due to a previously unrecognized mechanism characterized by poor anticoagulant response to activated protein $\mathrm{C}$ : prediction of a cofactor to activated protein C. Proc Natl Acad Sci USA $1993 ; 90: 1004-8$.

43. Bertina RM, Koeleman BPC, Koster T Rosendaal FR, Dirven RJ, de Ronde H, van der Velden PA, Reitsma PH. Mutation in blood coagulation factor $\mathrm{V}$ associated with resistance to activated protein C. Nature $1994 ; 369: 64-7$

44. Haldane JBS. The relative importance of principal and modifying genes in determining some human diseases. I Genet 1941; $41: 149-57$

45. Férec C, Mercier B, Audrézet M. Les mutations de la mucoviscidose: du génotype au phénotype. médecine/sciences $1994 ; 10$. pe au pho

46. Dean M, Santis G. Heterogeneity in the severity of cystic fibrosis and the role of CFTR gene mutations. Hum Genet 1994; 93 . C.FTR

47. Kiesewetter S, Macek M Jr, Davis C, Curristin SM, Chu CS, Graham C, Shrimpton AE, Cashman SM, Tsui L, Mickle J, Amos J, Highsmith WE, Shuber A, Witt DR, Crysta RG, Cutting GR. A mutation in CFTR produces different phenotypes depending on chromosomal background. Nature Genet $1993 ; 5: 274-7$

48. Chu CS, Trapnell BC, Curristin S, Moss J, Dalemans W, Jallat S, Mercenier A, Pavirani A, Lecocq JP, Cutting GR, Guggino exon 9 skipping in cystic fibrosis transmembrane conductance regulator mRNA. Nature Genet 1993; 3: 151-6.

49. Wolf $\mathrm{U}$. The genetic contribution to the phenotype. Hum Genet 1995; 95 : 127-48.

50. Valentijn LJ, Baas F, Wolterman RA Hoogendijk JE, van den Bosh NHA, Zorn I, Gabreëls-Festen AAWM, de Visser M, Bolhuis P. Identical point mutations of PMP-22 in Trembler-J mouse and Charcot-MarieTooth disease type 1A. Nature Genet $1992 ; 2$ : 288-91.

51. Rutland P, Pulleyn LJ, Reardon W, Baraitser M, Hayward R, Jones B, Malcolm S, Winter RM, Oldridge M, Slaney SF, Poole $\mathrm{MD}$, Wilkie AOM. Identical mutations in the FGFR2 gene causes both Pfeiffer and Crouzon syndrome phenotypes. Nature $G e-$ net $1995 ; 9: 173-6$
Si les $\alpha$-thalassémies délétionnelles sont fréquentes en Afrique et plus encore au Moyen Orient ou en Inde, on n'en retrouve pas chez les drépanocytaires du Bassin méditerranéen. Ce fait a été constaté dans le nord de la Grèce [29]. En Algérie aussi, il était surprenant de ne pas en trouver, alors qu'on observe une fréquence relativement élevée de gènes $\beta^{s}$ ou $\beta$-thalassémiques, et que le pays a connu des siècles d'endémie palustre; un criblage néonatal montrait, par ailleurs, une incidence non négligeable (10$15 \%$ ) d'Hb Bart $\left(\gamma_{4}\right)$, témoin d'une production insuffisante de chaîne $\alpha$. Une étude systématique récente par des techniques d'électrophorèse en gradient de dénaturation (DGGE) ou effectuées après dénaturation (SSCP) a montré la fréquence et la diversité de formes d' $\alpha$-thalassémie non délétionnelle qui ont pu jouer le même rôle que les formes délétionnelles au cours de l'évolution.

\section{Maladie drépanocytaire et régulation cellulaire de la production $d^{\prime} H b F$}

Les deux chapitres précédents ont traité d'aspects moléculaires concernant le rapport relatif d'HbF et d'HbS et la concentration d'hémoglobine dans le globule rouge. Il existe aussi un aspect cellulaire à la production d'HbF dans la mesure où celle-ci est limitée à un groupe particulier de cellules érythroïdes : les cellules F. Cet aspect est important car plus fort est le pourcentage de cellules $\mathrm{F}$, plus grand est le nombre de cellules protégées de la falciformation. En fait, le taux d'HbF tel qu'on l'apprécie chez un sujet drépanocytaire est le résultat de trois composantes distinctes: le nombre de cellules $F$, la quantité d'HbF par cellule $F$, enfin, la survie sélective des cellules $\mathrm{F}$ comparée à celle des cellules ne contenant pas d'HbF. Cette dernière composante est liée à la physiopathologie de la maladie, les deux premières en revanche sont génétiquement déterminées. Le taux d'HbF par cellule F serait sans doute, comme nous l'avons vu plus haut, associé $\mathrm{au}(\mathrm{x})$ polymorphisme(s) du locus $\beta$. Le pourcentage de cellules $\mathrm{F}$ semble, au contraire, contrôlé par un déterminant agissant en trans. Différentes hypothèses ont été proposées concernant la localisation du déterminant en cause.

Étudiant le nombre de cellules $\mathrm{F}$ dans une population normale, non anémique, un groupe japonais a suggéré que ce déterminant serait lié au chromosome X [30]. Une hypothèse similaire a été formulée à partir de l'étude de 292 adultes drépanocytaires jamaïcains; par analyse de liaison avec une série de polymorphismes, le déterminant a même été localisé en Xp22.2 [31]. Dans un travail plus récent explorant à nouveau une cohorte de 257 drépanocytaires jamaïcains, la même équipe a présenté une étude statistique tenant compte de variables multiples (âge, sexe, nombre de gènes $\alpha$, haplotypes). En conclusion de ce travail, le déterminant lié au chromosome $\mathrm{X}$ est donné comme responsable d'environ $40 \%$ des variations observées du taux d'HbF [32].

La démonstration de l'existence d'autres déterminants participant à la régulation du taux des cellules $\mathrm{F}$ vient d'être apportée par une recherche totalement différente menée par un groupe anglais sur une très grande famille indienne (4 générations, 166 sujets) présentant une persistance héréditaire d'Hb F. Dans cette famille, la ségrégation du caractère PHHF est indépendante du chromosome $\mathrm{X}$ et du locus $\beta$-globine, sur le chromosome 11 , mais liée à des marqueurs sur le bras long du chromosome $6[33,34]$. Une recherche menée récemment par notre groupe sur des familles siciliennes et permettant l'exploration, non seulement de sujets SS, mais des hétérozygotes AS dans les mêmes familles, nous oriente également vers une transmission autosomique indépendante du locus $\beta$-globine. Ces résultats pourraient ne pas être incompatibles avec les précédents: d'une part, ils ne concernent pas les mêmes populations, mais, surtout, il semblerait que, comme d'autres données quantitatives, le pourcentage de cellules $\mathrm{F}$ soit déterminé par un contrôle multifactoriel.

\section{Maladie drépanocytaire, facteurs cellulaires et rhéologiques}

Certaines complications de la maladie drépanocytaire ne sont de toute évidence pas la conséquence directe 
du processus de polymérisation. Si l'on peut en effet imaginer que les accidents de la microcirculation sont directement dus à une obstruction par des cellules rigidifiées, il ne peut en être de même quand il y a infarctus des gros vaisseaux. Depuis de nombreuses années déjà on parle d'une maladie rhéologique [35], mais ce n'est que relativement récemment que cet aspect focalise l'attention des chercheurs. Il concerne les interactions du globule rouge drépanocytaire avec son environnement. De nombreux facteurs interviennent sûrement à ce niveau. On sait que la polymérisation intra-érythrocytaire de l'HbS s'accompagne de désordres de la membrane touchant les phospholipides et les protéines et aboutissant à l'élimination de microvésicules. L'ap-

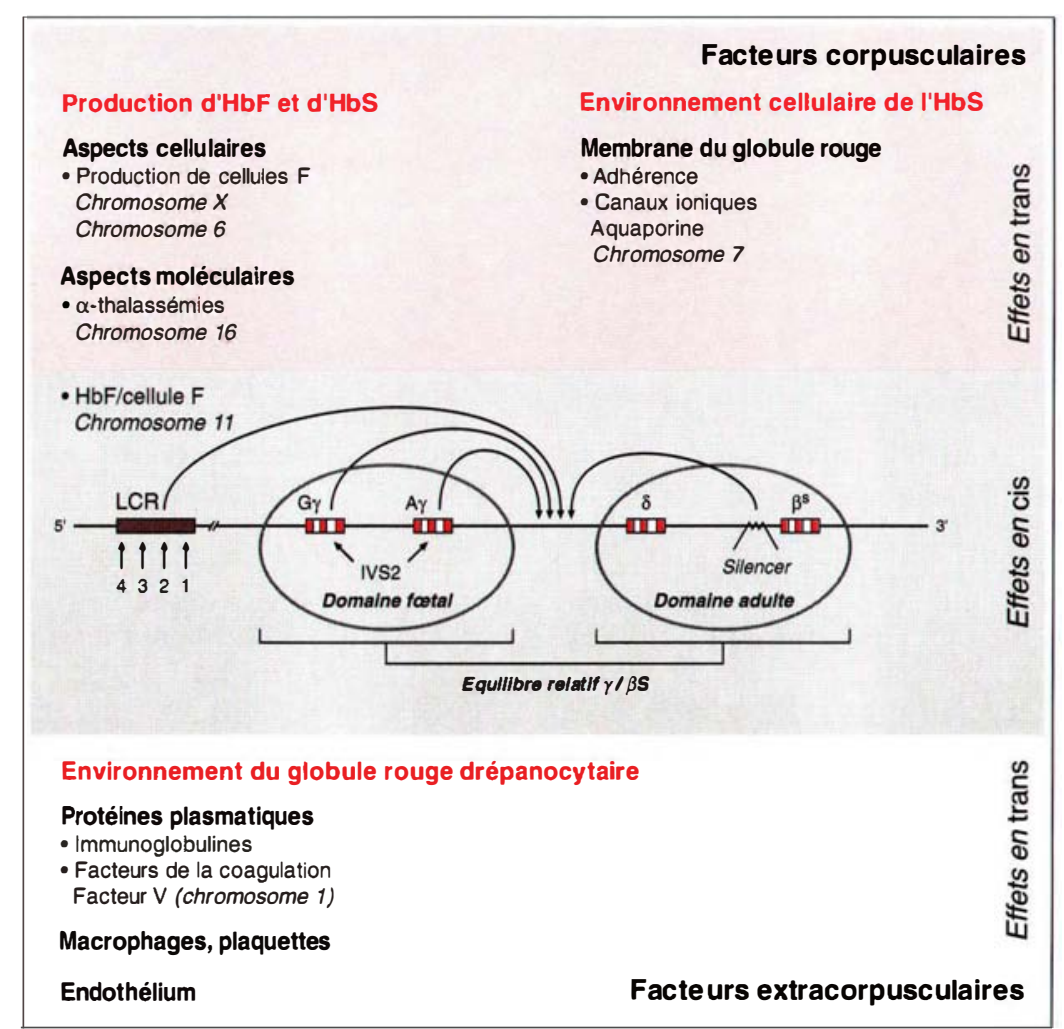

Figure 3. Déterminants susceptibles de moduler le processus physiopathologique de la drépanocytose. On distinguera les facteurs corpusculaires et les facteurs extracorpusculaires. Dans le premier groupe, certains déterminants sont situés en cis du gène $\beta$; ce sont les polymorphismes du locus $\beta$-globine, détaillés dans la figure 2, et qui modulent l'équilibre $d^{\prime}$ expression $\gamma \beta^{S}$ et donc le taux $d^{\prime} \mathrm{Hb}$ F/cellule $F$. De nombreux autres facteurs corpusculaires sont situés en trans du locus des gènes de la famille $\beta$ globine; ils incluent le nombre de gènes $\alpha$ fonctionnels (existence $d$ 'une $\alpha$-thalassémie associée), les déterminants génétiques du contrôle de la production des cellules $F$ et les gènes des protéines membranaires du globule rouge. Le groupe des facteurs extracorpusculaires est aussi multiple; il concerne l'environnement protéique et cellulaire du globule rouge drépanocytaire, et inclut les protéines sulte d'un mécanisme différent dans la mesure où elle est le fait des cellules jeunes, abondantes en réponse à l'hémolyse. Il semble que la protéine en cause sur la membrane soit le complexe des intégrines $\alpha 4 \beta 1$ ou VLA-4, qui se lie à la molécule d'adhérence des cellules vasculaires VCAM-1 dont l'expression est sous l'influence des cytokines $\left(\mathrm{m} / \mathrm{s} n^{\circ} 4\right.$, vol. 11, p. 633) [40].

Qu'ils soient primitifs ou secondaires, les troubles rhéologiques font intervenir des protéines du globule rouge ou des protéines partenaires qui peuvent, elles aussi, être polymorphes. Par exemple, on a récemment décrit un polymorphisme Cys $\rightarrow$ Ser sur une protéine de membrane, l'aquaporine CHIP28 (m/s $n^{\circ} 3$, vol. 9 , p.334), susceptible de modifier la perméabilité érythrocytaire à l'eau et d'intervenir dans l'état d'hydratation du globule rouge drépanocytaire [41]. Le gène correspondant est localisé sur le chromosome 7. En termes de protéines partenaires, on peut évoquer les protéines de la coagulation. Une voie active d'investigation concerne la recherche d'un état génétique de prédisposition aux thromboses chez les drépanocytaires victimes d'accidents vaso-occlusifs des gros vaisseaux. En effet, à côté des déficits génétiques rarissimes en protéine $\mathrm{C}$ et protéine $\mathrm{S}$, on a montré récemment l'existence d'un polymorphisme du facteur $\mathrm{V}$, substrat de la protéine $\mathrm{C}$ activée, fréquent chez les Européens (Arg $\rightarrow \mathrm{Gln}$ en position 506); il est responsable d'une augmentation de dix fois du risque thrombotique chez l'adulte $\left(m / s n^{\circ} 2\right.$, vol.1 0, p. 231; $n^{\circ} 6-7$, vol.1 0, p. 733) $[42,43]$. Le gène du facteur $V$ est localisé en 1q. Il est facile d'imaginer un phénomène identique ou similaire à l'origine des thromboses des gros vaisseaux chez les drépanocytaires.

La figure 3 résume l' ensemble des facteurs identifiés à ce jour comme susceptibles d'interagir avec le mécanisme physiopathologique primaire de la drépanocytose. La liste n'est sûrement pas exhaustive, et on peut facilement concevoir que de tels polymorphismes modifient l'apparition ou l'évolution de complications de la maladie. Il est évident que, dans tous les cas, l'influence de ces différents facteurs sera plus facile à étudier sur 
des populations bien déterminées, dont le capital génétique est relativement homogène, que sur les cas individuels de populations totalement mêlées.

\section{Conclusion}

Nous avons vu l'influence que peuvent exercer des polymorphismes multiples sur l'expression d'une maladie monogénique apparemment très bien connue. Il en est, ou en sera, sans doute de même concernant d'autres maladies monogéniques. Dès 1941, Haldane évoquait l'intervention de gènes modificateurs dans les maladies mendéliennes [44]. Le cas de la mucoviscidose est particulièrement intéressant, puisqu'il s'agit de la maladie monogénique la plus fréquente dans nos populations européennes [45]. Elle présente cependant avec la drépanocytose des différences importantes: le gène concerné, $C F \Gamma R$, est de beaucoup plus grande taille; les mutations identifiées à ce jour sont nombreuses, même si l'une d'entre elles, la délétion de la phénylalanine 508 $(\Delta \mathrm{F} 508)$, est en Europe largement prédominante. La mucoviscidose est d'expression pluritissulaire et le polymorphisme génétique peut s'y exprimer par une atteinte variable des différents tissus. Parmi les deux signes majeurs de la maladie, l'insuffisance pancréatique plus ou moins complète et ses conséquences semblent pouvoir être assez bien corrélées au génotype; en revanche, la plus grande variabilité est observée concernant les manifestations respiratoires chez des sujets par ailleurs porteurs de la même mutation du gène $C F T R$. Cela fait évoquer le rôle d'autres facteurs, liés à l'environnement mais aussi génétiques [46]. Comme dans la drépanocytose, certains facteurs sont situés en cis, dans le gène CFTR lui-même, mutation correctrice ou épissage alternatif entraînant l'élimination de

TIRÉS À PART

D. Labie certains exons [47]. On a, en particulier, évoqué le rôle de régulation quantitative exercé par la longueur variable du tractus polypyrimidique dans le site 3' d'épissage précédant l'exon 9 qui peut être excisé [48]. D'autres se situeraient en trans du gène de la mucoviscidose, et, puisqu'il s'agit d'infection respiratoire chronique, on a évoqué les déterminants contrôlant une réponse humorale ou cellulaire à des antigènes ou une réponse inflammatoire, molécules HLA, récepteur d'interleukine, $\mathrm{TNF} \alpha$, ainsi que l'activité potentielle d'autres canaux chlorure [46].

Bien que moins étudié, le même phénomène de variabilité clinique dans le cas de mutation identique a été largement signalé dans d'autres affections [49]. La maladie de CharcotMarie Tooth de type 1A en est un exemple $\left(\mathrm{m} / \mathrm{s} n^{\circ} 11\right.$, vol.9, p. 1273) $[50]$. Un autre exemple très récemment décrit est celui de la même mutation du gène FGFR2 pouvant, selon les cas, évoluer vers un syndrome de Pfeiffer ou vers un syndrome de Crouzon $\left(m / s n^{\circ} 12\right.$, vol. 11, p. 1748) [51]. Action épistatique due à un autre déterminant, variation au cours du développement ou variabilité de spécificité tissulaire, les causes sont multiples qui permettent d'envisager beaucoup de maladies monogéniques comme des maladies à modulation multifactorielle

\section{Remerciements}

Les auteurs remercient très vivement Rajagopal Krishnamoorthy pour toutes les discussions particulièrement enrichissantes qu'ils ont pu avoir avec lui concernant le sujet.

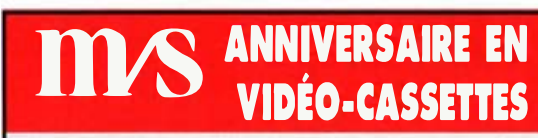

Les conférences de la journée du $10^{\circ}$ anniversaire de médecinel sciences du 16 mars 1995 sont disponibles sur vidéo-cassettes auprès de :

ASSOCIATION DIFFUSION

DES CONNAISSANCES

2, avenue Léon-Bernard, 35043 Rennes Cedex, France.

\section{Summary}

Sickle cell anemia: an example of the polygenic modulation of monogenic diseases

Variability in the clinical expression of many monogenic diseases has always been very puzzling. Because sickle cell anemia results from a single mutation, and because its pathophysiological mechanism has been thoroughly investigated, it constitutes a model of choice to investigate this issue and can bring already some informative clues. Three features of the pathophysiological scheme can be the target of modifying phenomena: the process of hemoglobin $\mathrm{S}$ ( $\mathrm{Hb} \mathrm{S})$ polymerization, the intraerythrocytic concentration of $\mathrm{Hb} \mathrm{S}$, and an expression profile of proteins, other than hemoglobin, specific to reticulocytes in sicklers. Factors that can modify each of these three features have been characterized. (1) Polymerization can be hindered by other hemoglobins, mainly fetal hemoglobin (Hb F). (2) Intraerythrocytic concentration of $\mathrm{Hb} \mathrm{S}$ is decreased by a coinherited $\alpha$-thalassemia or by a modulation of the action of transacting factors on the respective expression of the fetal $\gamma$-genes vs the adult $\beta$-gene. (3) Sickle cell anemia has also been defined as a cellular and rheological disease, various mechanisms of which have been studied: membrane modifications, increased adhesion to the vascular endothelium, activation of the coagulation cascade. All these processes involve partner proteins that are or could be polymorphic. Genes coding for these proteins can be located in cis from the $\beta^{\text {s }}$ gene or in trans on other chromosomes. Their combined action can be described as the polygenic control of this monogenic disease. 